\title{
Administration of Cilostazol, an Antiplatelet, to Patients with Acute-stage Cerebral Infarction and Its Effects on Plasma Substance P Level and Latent Time of Swallowing Reflex
}

\author{
Arata Abe, Yasuhiro Nishiyama, Hiroshi Hagiwara, Seiji Okubo, \\ Masayuki Ueda, Ken-ichiro Katsura and Yasuro Katayama \\ Divisions of Neurology, Nephrology, and Rheumatology, Department of Internal Medicine, Nippon Medical School
}

\begin{abstract}
Background and Objective: It has been reported that medical treatment with cilostazol (cilo) as an antiplatelet may increase a substance $\mathrm{P}$ level in the striatum to shorten the latent time of swallowing reflex (LTSR). We undertook a pilot study to confirm whether cilo administration to patients with cerebral infarction is effective in increasing their plasma substance P level and then in ameliorating the status of LTSR.

Methods and Subjects: Eligible subjects were recruited, after informed consents, from 20 hospitalized patients with acute-phase cerebral infarction within 72 hours from the onset. At the start of treatment, the subjects were assigned at random to those given aspirin alone (noncilo group) and those given aspirin plus cilo (cilo group). Plasma substance P levels and LTSR values were measured at the starting point (baseline), 28 days after, and 180 days after.

Results and Discussion: No significant time-dependent change in plasma substance $\mathrm{P}$ level was found probably because of large individual differences but, 28 days after the start of treatment, this value tended to become higher in cilo group than in non-cilo group $(\mathrm{P}<0.10)$. Whereas, in terms of fold changes of LTSR in cilo group, there was a significant between-term difference at $\mathrm{P}<0.05$, indicating that this medication is effective in ameliorating the swallowing function is improved in the long run.

Conclusion: The LTSR values was significantly shortened within 180 days after the start of cilo treatment, but the result was not well explained by substance P levels as far as these were measured using the plasma, probably because this substance had diluted during blood circulation. However, it will become clinically usable as a single swallowing index, if in the future some ingeneus method of its measurement is developed. A larger-scale study would also be needed to confirm our conclusion from this pilot study.
\end{abstract}

(J Nippon Med Sch 2013; 80: 50-56)

Key words: substance P, Cilostazol Stroke Prevention Study (CSPS), simple swallowing provocation test, acute cerebral infarction, latent time of swallowing reflex

Correspondence to Arata Abe, MD, PhD, Department of Neurological Science, Graduate School of Medicine, Nippon Medical School, 1-1-5 Sendagi, Bunkyo-ku, Tokyo 113-8603, Japan

E-mail: abe@nms.ac.jp

Journal Website (http://www.nms.ac.jp/jnms/) 


\section{Introduction}

Stroke still remains a major health problem worldwide. Patients with stroke confront high risks of specific medical complications such as psychological and neurological disorders, infections, thromboembolism, and pains ${ }^{1}$. Among them, pneumonia occurs as one of the most serious complications in approximately $22 \%$ of patients after the onset of stroke $^{2}$, and influences the patient's subsequent prognosis ${ }^{3}$. It was reported that cilostazol (cilo) as an antiplatelet drug controlled the onset of geriatric aspiration-associated pneumonia caused by cerebral infarction ${ }^{4}$ and its own preventing effect on recurrent cerebral infarction can be inferred from as a result of a subgroup analysis in the large-scale clinical study (Cilostazol Stroke Prevention Study: CSPS) ${ }^{3-5}$. Zhang et al. ${ }^{6}$ have carried out an experiment with chronic cerebral hypoperfusion model rats and suggested that cilo could be protective against pneumonia at a chronic stage of stroke; this may be based on its potencies to modulate dopamine and substance $\mathrm{P}$ levels in the striatum for improved swallowing reflex. A mechanism of improving the swallowing function by cilo may also be its inhibition of decreasing dopamine and substance $\mathrm{P}$ levels through activating the intracellular signal transmission system via phosphorylation of cyclic adenosine monophosphate responsive element binding protein $(\mathrm{CREB})^{7-9}$. Moreover, papers ${ }^{10,11}$ have described that cilo improved the LTSR in post-stroke patients by increasing their plasma substance $\mathrm{P}$ level, while no such effect was found in aspirin used together.

Because angiotensin converting enzyme (ACE) inhibitors have been reported to be efficacious in prevention of aspiration-associated pneumonia by increasing a substance $\mathrm{P}$ level, cilo may also have the same mechanism to prevent the pneumonia caused by cerebral infarction ${ }^{11-13}$. However, no information is available regarding the changes in substance $\mathrm{P}$ level in patients with acute-stage cerebral infarction and there has been no paper on an effect of cilo to increase the substance $\mathrm{P}$ level in patients during hospitalization. In the present study, we evaluated the influence of cilo treatment on plasma substance $\mathrm{P}$ levels and LTSR values in patients with acute-stage cerebral infarction by a randomized open-label controlled method.

This study purposes confirming whether there is a correlation between substance $\mathrm{P}$ level and swallowing function in patients with cerebral infarction. In detail, cilo-treated patients with an acute-stage cerebral infarction were investigated for time-dependent changes in their plasma substance $\mathrm{P}$ level and latent time by use of Simple Swallowing Provocation Test (S-SPT) as abbreviated LTSR ${ }^{11,14,15}$.

\section{Subjects and Methods}

Patients with acute-phase cerebral infarction (excluding cardiogenic cerebral embolism), who could receive anti-platelet test drugs within 72 hours after the onset of cerebral infarction were recruited as subjects eligible for randomization treatment. They were enrolled in this clinical study between August 2008 and March 2009.

Patients were excluded from this study in the following cases: (1) having swallowing difficulty due to dysphagia before the onset of cerebral infarction at a food aspiration difficulty level of less than or equal to the clinical severity classification criteria $^{17}$; (2) prescribing ACE inhibitors or capsaicin with an influence on substance P level ${ }^{11-13}$; (3) having cerebral bleeding or a tendency of intracerebral hemorrhage; and (4) gastrointestinal ulcer or congestive heart failure. Other exclusion criteria included the case of hypersensitivity or anaphylaxis to test drugs administered, and also included women who were pregnant or child-bearing and so judged to be inappropriate for the enrollment.

The study was approved by the Ethical Committee of this hospital. Written informed consents were obtained from all the patients after the study protocol had been fully explained prior to study participation.

Twenty-one inpatients with cerebral infarction were found to meet all these inclusion criteria, with no exclusion criterion included in this randomized open-label controlled study. Eligible subjects were randomized to the two treatment groups: the cilo 


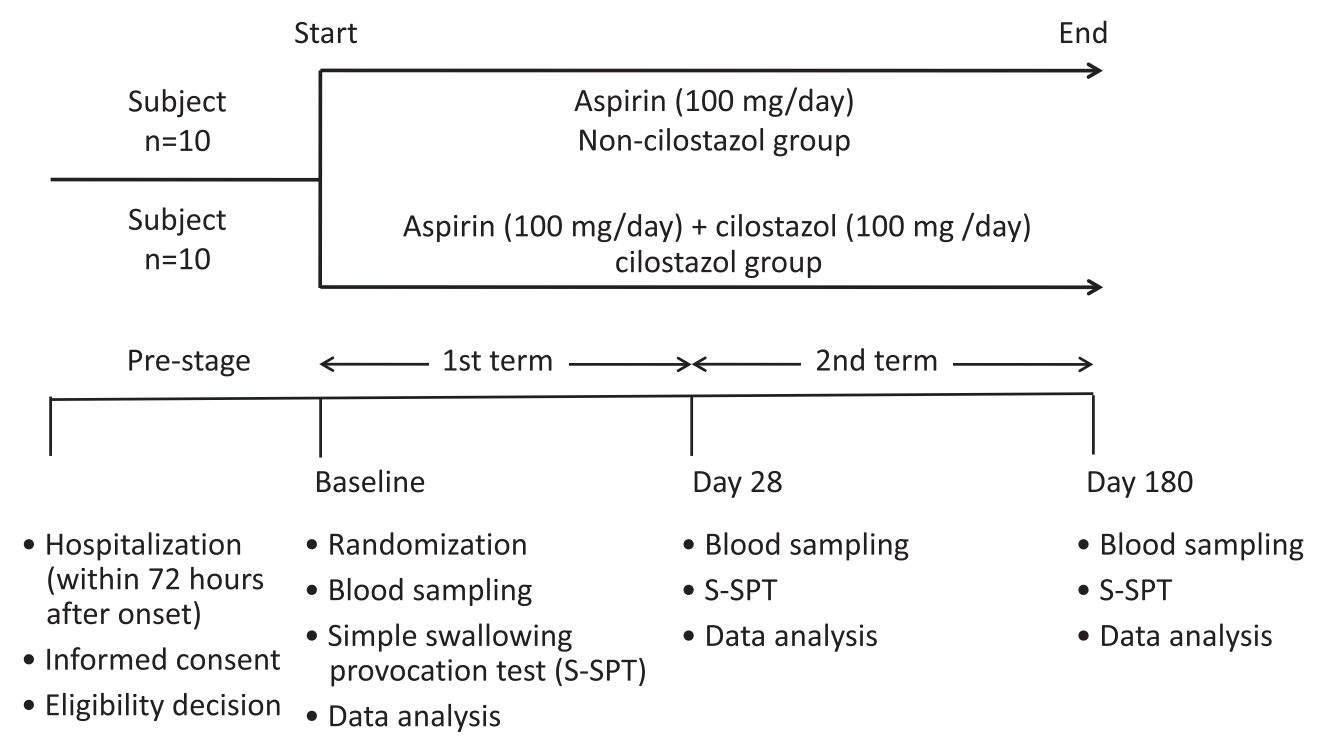

Fig. 1 Study design.

group in which each one was orally given cilo at 100 $\mathrm{mg} /$ day and aspirin $100 \mathrm{mg}$ /day concominatly for 180 days and the non-cilo group in which each was given aspirin alone at $100 \mathrm{mg} /$ day similarly. The outline of this study protocol is shown in Figure $\mathbf{1}$. By the way, the combination use of other medications, e.g. tissue plasminogen activator, edaravone, argatroban hydrate, and ozagrel sodium, were not specified to be excluded during this study. The names of concomitant drugs and the periods of their use were recorded.

For plasma substance $\mathrm{P}$ measurement, blood samples were collected and the plasma fractions were taken. Each of $250 \mathrm{~mL}$ aliquots was dispersed in a designated holder containing an additive for stabilization, prior to freezing and storing below $-40^{\circ} \mathrm{C}$. Frozen samples were sent to SRL, Inc. (Tokyo, Japan) within 2 weeks after their preparation. The samples were then kept below $-70^{\circ} \mathrm{C}$ and measured for substance $\mathrm{P}$ by use of monoclonal antibody ${ }^{11}$. On the other hand, LTSR in each subject was induced by bolus injection of 0.4 $\mathrm{mL}$ distilled water into oropharynx from nasal cavity through a 5-Fr small nasal catheter at the supine position $^{11}$. LTSR values were obtained during the time from the injection to the onset of swallowing reflex. This procedure was conducted twice at the interval of 1 minute. The results were presented as fold changes, i.e., LTSR values on 28 or 180 days divided by those at baseline, and shown as means \pm standard deviations.

\section{Statistics}

Baseline characteristics and demographics of patients are shown Table 1. The qualitative baseline data tabulated were contrasted using Fisher's exact test and shown as median values \pm their biases obtained by Mann-Whitney test. Also, between-term differences and between-group differences in plasma substance P level and those in fold change of LTSR (Fig. 1) were analyzed by Student's $t$-test, with their statistical significance considered at $\mathrm{P}<0.05$. Incidentaly, we also used the data after stratification of the subject according to whether their LTSR values were $>10$ seconds or $\leq 10$ seconds in consideration of the reports presented by Teramoto et $\mathrm{al}^{11,14,15}$, and analyzed the stratified data for statistical significance.

\section{Results}

When the National Institute of Health: Stroke Scale (NIHSS) of the non-cilostazol group and that of the cilostazol group were compared in terms of facial palsy as well as palsies of upper and lower extremities at baseline, no significant between-group difference was found in mean \pm standard deviation: 
Cilostazol Improves Swallowing

Table 1 Baseline characteristics in patients

\begin{tabular}{lcc}
\hline \multicolumn{1}{c}{ Characteristic } & $\begin{array}{c}\text { Non-cilostazol group } \\
(\mathrm{n}=10)\end{array}$ & $\begin{array}{c}\text { Cilostazol group } \\
(\mathrm{n}=10)\end{array}$ \\
\hline $\begin{array}{l}\text { Demographics } \\
\text { Male/female }\end{array}$ & $4 / 6$ & $6 / 4$ \\
Age, years ${ }^{1)}$ & $74(53-85)$ & $65(48-80)$ \\
Current smoker & 3 & 3 \\
$\quad$ Subtype of cerebral infarction & $(6,4)$ & $(7,3)$ \\
(lacunar infarction, atherosclerosis) & & $(6,1,2,1)$ \\
$\quad$ Modified Rankin Scale (0, 1, 2, 3) & $(8,2,0,0)$ & 3 \\
Complications/past histories & & 3 \\
Hypertension & 6 & 5 \\
Diabetes mellitus & 1 & \\
Dyslipidemia & 6 & \\
\hline
\end{tabular}

1)Shown as median values with their ranges.

No between-group difference detected at $\mathrm{P}<0.05$ in any characteristic by Fisher's

exact test or Mann-Whitney test., or both.

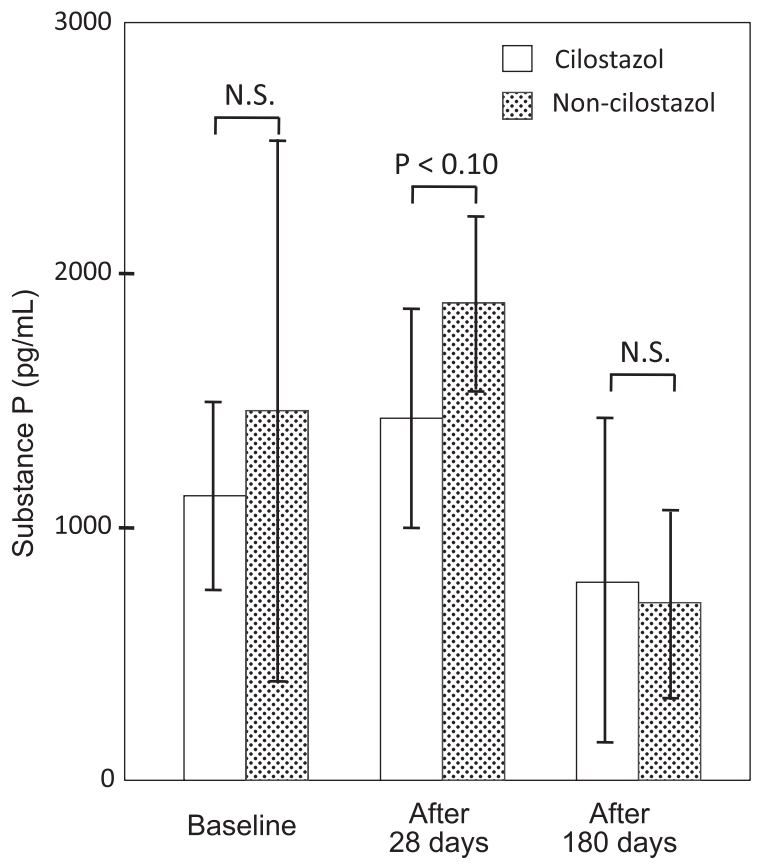

Fig. 2 Time-dependent comparison between the groups of plasma levels of substance P.

$2.5 \pm 1.9$ vs $2.5 \pm 1.6$. To evaluate the effect of cilo treatment of all the subjects $(n=14)$ on their plasma substance $\mathrm{P}$ level, clinically eligible subjects $(n=14)$ out of all the recruited ones $(n=20)$ were assigned at random. This randomization was found appropriate, since the mean value in the cilo group and that in the non-cilo group were almost similar to each other at baseline (Fig. 2). At the endpoint of the first term, i.e., 28 days after the start (Fig. 1), there was the tendency that the substance $\mathrm{P}$ level in the cilo group became higher than that in the non-cilo group $(\mathrm{P}<$ 0.10 ), with the result that the occurrence of a little, though not statistically significant, effect of cilo administration on substance $\mathrm{P}$ induction. However, the effect was mitigated at the endpoint of the second term, i.e., 180 days after (Fig. 1) for some unidentified reason. By the way, we failed to find any between-term significance because of large individual differences in terms of standard deviations of mean values. Even the statistical analysis applied to the two stratified subjects with LTSR $>10$ and LTSR $\leq 10$ gave no significant between-group difference (data not shown).

Next, fold changes in LTSR was analyzed, with the result that, although there was no significant between-group difference, a significant betweenterms difference $(\mathrm{P}<0.05)$ was found in the case of the cilo group (Fig. 3). Incidentally, the distinct substance $\mathrm{P}$ decreases in both group (Fig. 2) may be due to a natural process taking place over period of 180 days; the substance $\mathrm{P}$ decreasing effect of cilostazol remains unclear because it was used at the half amount. Also, the LIST was safely conducted all the way; neither cerebral hemorrhage nor microbleeds occurrence was observed.

\section{Discussion}

It is likely that plasma substance $\mathrm{P}$ levels in patients after the onset of cerebral infarction vary 


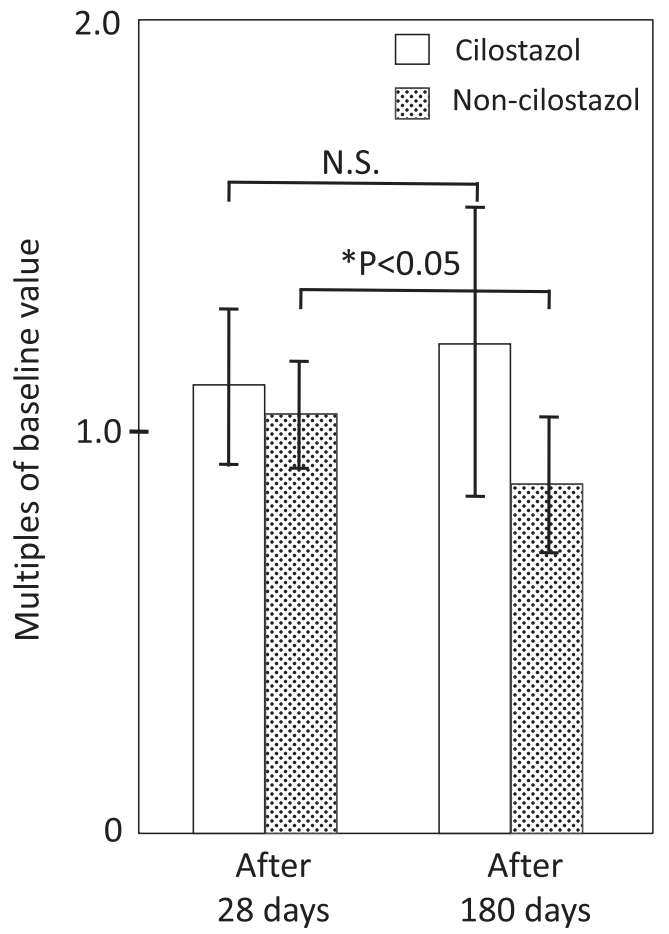

Fig. 3 Time-dependent decrease in latent time of the swallowing reflex (LTSR).

*: significant; and N.S.: not significant

depending on many factors and thus their swallowing functions change accordingly. Capsaicin and ACE inhibitors as dopaminergic agonists induce substance $P$ through dopamine supplementation ${ }^{11-13,19-22}$ and prevent aspiration pneumonia as a complication of cerebral infarction ${ }^{1,219}$. Biochemically, substance $\mathrm{P}$ is synthesized in cervical nerve clause of a vagus nerve sensory branch and then released to reach pharynx and trachea. It may be essential to keep swallowing function and cough reflex normal via adjustment by the dopamine nervous system in basal ganglia. It deserves to note that cilo effectively improves swallowing function by activation of tyrosine hydroxylase through striatal CREB phosphorylation in the case of chronic cerebral hypoperfusion model rats whose substance $\mathrm{P}$ level and LTSR values differing from our observations

(Fig. 2 and Fig. 3). Which probably means that the effect of the increase in substance $P$ level on day-28 remains to reduce the LTSR value on day-180. This suggests that the plasma substance P level may not directly reflect any genuine value of this compound in the brain. Using a method of S-SPT, Teramoto et al. ${ }^{11,14,15,28}$ measured LTSR and defined it's critical value at 3 seconds; their studies employed only male patients with non-cardiogenic embolism in 1-6 months from the onset of symptom. They also used cilo at $200 \mathrm{mg} /$ day. Whereas, we used it at $100 \mathrm{mg} /$ day concomitantly in combination with $100 \mathrm{mg} /$ day aspirin (Fig. 1), providing new data on the effect of cilo administration. Considering that the use of the two drugs in combination may be a risk factor for cerebral hemorrhage ${ }^{29}$, we used cilostazol at the half amount (100 mg/day) instead of using it at a normal level $(200 \mathrm{mg} /$ day). There are often issues to be considered according to Guidelines for the Management of Community-Acquired Pneumonia in Adults $^{30}$, evaluation of dysphagia involves water swallowing test, repetitive saliva swallowing test ${ }^{31}$, and simple swallowing provocation test ${ }^{32}$ as simple, conventional radiography-videofluoroscopic swallow examination $^{33,34}$, nasopharyngeal fiberscopescintigraphy as more detailed examinations. Although they are already established laboratory procedures, no information is an available regarding a standard value of latent time. Asymmetric dimethyl arginine may be another factor of interest which is an endogenic nitric monoxide synthase inhibitor causing vascular endothelium dysfunction $^{35,36}$. In considering all these factors related to swallowing function, it is important to put forward the effect of cilo as one of potential LTSR improves to gradually elicit a swallowing refleximproving activity.

\section{Conclusions}

The LTSR value we observed in the present study was not distinctly correlated to the substance $\mathrm{P}$ level as far as it was measured with the plasma fraction of blood, not with the striatum as reported ${ }^{6}$, probably because this substance had diluted during the circulation before measurement, although the tendency existed that the level became higher in the cilo group than in the non-cilo group 28 days after the start of treatment. Since the full LTSR decrease was found in the cilo group 180 days after the start, the plasma substance $\mathrm{P}$ level may be clinically usable as a simple swallowing reflex index on the 
condition that some ingenious method for measurement gets available in the future. Also, some larger-scale investigation would confirm our conclusion from this pilot study.

\section{References}

1. Langhorne P, Stott DJ, Robertson L, et al.: Medical complications after stroke: a multicenter study. Stroke 2000; 31: 1223-1229.

2. Shinohara Y, Yoshimoto T, Fukuuchi Y, et al.: Japanese Guideline for the Management of Stroke 2004, 2004; pp 8 (in Japanese).

3. Shinohara Y: Antiplatelet cilostazol is effective in the prevention of pneumonia in ischemic stroke patients in chronic stage. Cerebrovascular diseases 2006; 22 : 57-60.

4. Yamaya M, Yanai M, Ohrui T, et al.: Antithrombotic therapy for prevention of pneumonia. J Am Geriatr Soc 2001; 49: 687-688.

5. Gotoh F, Tohgi H, Hirai S, et al.: Cilostazol stroke prevention study. J Stroke Cerebrovasc Dis 2000; 9: 147-157.

6. Zhang N, Miyamoto N, Tanaka R, et al.: Activation of tyrosine hydroxylase prevents pneumonia in a rat chronic cerebral hypoperfusion model. Neuroscience 2009; 158: 665-672.

7. Tanaka K: Alteration of second messengers during acute cerebral ischemia adenylate cyclase, cyclic AMP-dependent protein kinase, and cycric AMP response element binding protein. Prog Neurobiol 2004; 65: 173-207.

8. Lee JH, Park SY, Shin HK, et al.: Protective effects of cilostazol against transient focal cerebral ischemia and chronic cerebral hypoperfusion injury. CNS Neurosci Ther 2008; 14: 143-152.

9. Lonze BE, Ginty DD: Function and regulation of CREB family transcription factor in the nervous system. Neuron 2002; 35: 605-623.

10. Ebihara T, Ebihara S, Watando A, et al.: Effect of menthol on the triggering of the swallowing reflex in elderly patients with dyspagia. Br J Clin Pharmacol 2006; 62: 369-371.

11. Teramoto S, Yamamoto H, Yamaguchi Y, et al.: Antiplatlet cilostazol, an inhibitor of type III phosphodiesterase, improves swallowing function in patients with a history of stroke. J Am Geriatr Soc 2008; 56: 1153-1154.

12. Ohrui T: Preventive strategies for aspiration pneumonia in elderly disabled persons. Tohoku J Exp Med 2005; 207: 1: 3-12.

13. Yamaya M, Sasaki H: Etilogy, pathogenesis and management of senile inflammatory pulmonary diseases. Nihon Kokyuki Gakkai Zasshi 2002; 40: 3-10 (Article in Japanese).

14. Teramoto S, Matsuse T, Fukuchi Y, et al:: Simple two-step swallowing provocation test for elderly patients with aspiration pneumonia. Lancet 1999; 353: 1243.

15. Teramoto S, Matsuse T, Fukuchi Y: Decision-making for safe feeding after stroke. Lancet 2000; 356: 1352.

16. Barton CW, Hemphill JC 3rd: Cumulative dose of hypertension predicts outcome in intracranial hemorrhage better than American Heart Association Guidelines. Soc Acad Emerg Med 2007; 14: 695-701.

17. Baba M, Saitoh E: Evaluation of swallowing impairment. NICHIDOKU-IHO 2001; 46: 17-25 (Article in Japanese).

18. Martens-Lobenhoffer J, Bone-Boeger SM: Fast and efficient determination of arginine, symmetric dimethylarginine, and asymmetric dimethylarginine in biological fluids by hydrophilic-interaction liquid chromatography-electrospray tandem mass spectrometry. Clin chem 2006; 52: 488-493.

19. Smithard DG, O'Neill PA, Park C, et al: Complications and outcome after acute stroke. Stroke 1996; 27: 1200-1204.

20. Arai T, Sekizawa K, Ohrui H, et al.: ACE inhibitors and protection against pneumonia in elderly patients with stroke. Neurology 2005; 64: 573-574.

21. Teramoto S, Yamamoto H, Yamaguchi Y, et al.: ACE inhibitors prevent aspiration pneumonia in Asian, but not Caucasian, elderly patients with stroke. Eur Respir J 2007; 29: 218-220.

22. Ohkubo T, Chapman N, Neal B, et al.: Effect of an angiotensin-converting enzyme inhibitor-based regimen on pneumonia risk. Am J Respir Crit Care Med 2004; 169: 1041-1045.

23. Shinohara Y, Ogawa A, Suzuki N, et al.: Japanese Guideline for the Management of Stroke, 2009; pp 118-119 (in Japanese).

24. Lim JH, Woo JS, Shin YW: Cilostazol protects endothelial cells against lipopolysaccharide-induced apoptosis through ERK1/2- and P38 MARK -dependent pathways. Korean J Intern Med 2009; 24: 113-122.

25. Miyamoto N, Tanaka R, Zhang N, et al.: Crucial for pCREB signaling in the differentiation and survival of neural progenitors under chronic cerebral hypoperfusion. Neuroscience 2009; 162: 525-536.

26. Nakagawa T, Ohrui T, Sekizawa K, et al.: Sputum substance $\mathrm{P}$ in aspiration pneumonia. Lancet 1995; 345: 1447.

27. Hong KW, Lee JH, Kim KY, et al:: Cilostazol: Therapeutic potential against focal cerebral ischemic damage. Curr Pharm Des 2006; 12: 565-573.

28. Teramoto S: Novel preventive and therapeutic strategy for post-stroke pneumonia. Expert Rev Neurother 2009; 9: 1187-1200.

29. Toyoda K, Yasaka M, Iwade K, et al.: Dual antithrombotic therapy increases severe bleeding events in patients with stroke and cardiovascular disease. Stroke 2008; 39: 1740-1745.

30. The JRS Guidelines for the Management of Community-Acquired Pneumonia in Adults, 2008; pp 44-45 (in Japanese).

31. DePippo KL, Holas MA, Reding MJ: Validation of the 3-oz water swallow test for aspiration following stroke. Arch Neurol 1992; 49: 1259-1261.

32. Horner J, Massey EW: Silent aspiration following stroke. Neurology 1988; 38: 317-319.

33. Nakagawa $T$, Sekizawa $K$, Arai $H$, et al.: High incidence of pneumonia in elderly patients with basal ganglia infarction. Arch intern Med 1997; 157: 321324.

34. Singh S, Hamdy S: Dysphagia in stroke patients. Postgrad Med J 2006; 82: 383-391. 
A. Abe, et al

35. Napoli C, Sica V, de Nigris F, et al:: Sulfhydryl angiotensin-converting enzyme inhibition induces sustained reduction of systemic oxidative stress and improves the nitric oxide pathway in patients with essential hypertension. Am Heart J 2004; 148: e5.

36. Mamatha SN, Nagaraja D, Philip M, et al: Asymmetric dimethylarginine as a risk marker for early-onset ischemic stroke in Indian population. Clin Chim Acta 2011; 412: 139-142.

(Received, March 14, 2012)

(Accepted, September 11, 2012) 